\title{
Correlation between fragility and cooperativity in segmental dynamics of glass-forming para-substituted polystyrenes
}

\author{
Takashi Sasaki ${ }^{1}$, Mizuki Ichimura ${ }^{1}$ and Satoshi Irie $^{2}$
}

To understand the mechanism of the viscous slowdown in supercooled liquids that is responsible for glass transition, we investigate the interrelation between glass transition temperature $T_{\mathrm{g}}$, fragility and cooperativity in segmental dynamics. Polymeric glass formers having a similar chemical structure are expected to exhibit clear correlations between the above parameters. In this paper, polystyrene (PS) derivatives possessing various para-substituents are studied using calorimetry with regard to the fragility parameter $m$, dynamic length scale $\xi$ and the number of cooperatively rearranging segments $N_{\mathrm{CRR}}$ at $T_{\mathrm{g}}$. Positive correlations were revealed for both $N_{\mathrm{CRR}}\left(T_{\mathrm{g}}\right)$ vs $m$ and $\xi^{3}\left(T_{\mathrm{g}}\right)$ vs $m$. Both fragility and cooperativity were found to increase as the bulkiness of the substituent increases. Wide-angle X-ray scattering measurements revealed that the structural correlation between backbone chains is reduced as the bulkiness increases. This may be responsible for the reduced cooperativity. In contrast, for poly(methacrylic acid ester)s, the relations between the above parameters appeared to be less clear. The clear trend observed for the PS system may be due to the rigidity of the phenylene unit, through which the substituent directly affects the backbone dynamics. The estimated activation energy per segment increased consistently with an increase in the para-substituent's bulkiness.

Polymer Journal (2015) 47, 687-694; doi:10.1038/pj.2015.50; published online 8 July 2015

\section{INTRODUCTION}

The viscosity of supercooled liquids increases dramatically upon cooling, leading to vitrification. Such massive and continuous increase in viscosity is essential to the glass transition behavior, but its mechanism is not fully understood. ${ }^{1-4}$ One important feature is that the viscosity (or the relaxation time of segmental motions) depends on configurational entropy. This view is closely related to the picture of the cooperative rearrangement of segments (motional units). ${ }^{5}$ Here, the size of the cooperatively rearranging region (CRR) $\xi$ is a key parameter that characterizes segmental dynamics in supercooled liquids. The role of dynamic heterogeneity in segmental relaxation has also been investigated, ${ }^{3,6}$ and the size of cooperativity $\xi$ is often assumed to be directly connected to the length scale of the dynamic heterogeneity, $1,3,7,8$ though the relation between these lengths is still subject to much discussion. ${ }^{9}$ Based on the concept of segmental rearrangement within the CRR, the number of segments per CRR $N_{\mathrm{CRR}}$ is another essential parameter for characterizing the cooperative nature of segmental dynamics. ${ }^{5,10}$ In this study, for the purpose of convenience, we define $N_{\text {CRR }}$ as the number of repeating units per CRR.

The drastic viscous slowdown leads to a non-Arrhenius behavior that is characterized by dynamic fragility parameter $m$ defined as

$$
m=\left.\frac{\partial \log \tau}{\partial\left(T_{\mathrm{g}} / T\right)}\right|_{T=T_{\mathrm{g}}}
$$

where $\tau$ is $\alpha$ relaxation time and $T_{\mathrm{g}}$ is the glass transition temperature. ${ }^{11}$ The parameter $m$ is closely related to the effective activation energy at $T_{\mathrm{g}}$. The fragility $m$ becomes greater as nonArrhenius features become prominent. Many of the polymeric liquids are known to be fragile, that is, they exhibit relatively high values of $m \cdot{ }^{11,12}$ Experimental studies have revealed that both cooperativity and fragility depend significantly on polymer materials, that is, they are sensitive to detailed chemical structure. ${ }^{11-13}$ To elucidate the factors governing these parameters, it is essential to understand the mechanism behind viscous slowdown in polymer glass formers. It has been revealed that $m$ depends on the rigidity of backbone chains relative to the side group. ${ }^{13} \mathrm{Hu}$ et al. ${ }^{14}$ on the other hand, proposed an empirical method to predict the value of $m$ from the chemical structure of polymers. Furthermore, it is noteworthy that the fragility parameter plays an important role in determining the confinement effect on $T_{\mathrm{g} .}{ }^{15,16}$ This suggests that the origin of anomalous dynamics in nanoconfined polymeric systems is intimately related to the material dependence of fragility.

The relationship between fragility and cooperativity has been a crucial issue in elucidating the nature of the viscous slowdown behavior. One could reasonably anticipate that fragile glass formers would exhibit high cooperativity. However, in general, no clear positive correlation between $\xi$ and $m$ was reported for a wide variety of polymers. Interestingly, a series of poly( $n$-alkyl methacrylate)s were

${ }^{1}$ Department of Materials Science and Engineering, University of Fukui, Fukui, Japan and ${ }^{2}$ Department of Frontier Fiber Technology and Science, University of Fukui, Fukui, Japan

Correspondence: Professor T Sasaki, Department of Materials Science and Engineering, University of Fukui, 3-9-1 Bunkyo, Fukui 910 8507, Japan.

E-mail: sasaki@matse.u-fukui.ac.jp

Received 9 April 2015; revised 21 May 2015; accepted 1 June 2015; published online 8 July 2015 
found to show an apparent correlation between $\xi\left(T_{\mathrm{g}}\right)$ and $m \cdot{ }^{17}$ In this system, the backbone chemical structure was the same and only the length of the end alkyl group was systematically varied. Considering such a result, the relation between cooperativity and fragility is expected to show a rather simple trend for polymeric systems possessing similar chemical structures.

In this study, we investigate the interrelation of glass transition temperature, fragility and cooperativity parameters on a series of polystyrene (PS) derivatives with various para-substituents. It has been reported that the position of the substitution in the phenyl unit affects the backbone dynamics, and that para-substitution exhibits the strongest effect on $T_{\mathrm{g}}$ in polychlorostyene. ${ }^{18}$ As these polymers possess an identical backbone structure, we expect a systematic trend between the glass transition parameters. We discuss how the observed correlations between the parameters should be interpreted based on the entropy-based relaxation model. For comparison, we also present the results of poly(methacrylic acid ester)s (PMAEs). These polymers also possess various substituents as the end ester parts but their backbone structure is the same.

To evaluate cooperativity in segmental dynamics, several techniques have been developed until now, including four-point dynamic susceptibility, ${ }^{2,19-21}$ four-dimensional nuclear magnetic resonance, ${ }^{8,21}$ boson peak spectroscopy ${ }^{7,22,23}$ and the calorimetric method. ${ }^{17,19,24-26}$ Among these, calorimetry is a notably useful technique that can provide information on both fragility and cooperativity. In particular, for the evaluation of fragility, calorimetry is one of the most favorable methods for determining $m .{ }^{16}$ Furthermore, recent developments in temperature-modulated differential scanning calorimetry (DSC) allow one to obtain a rather broad range of the heat capacity spectrum. ${ }^{26}$ Here we employ DSC as an effective tool for the evaluation of both fragility and cooperativity, also allowing us to compare literature data on PMAEs obtained by the same technique. ${ }^{17,27}$

\section{EXPERIMENTAL PROCEDURE}

The polymers used in this study are listed in Table 1. They were all purchased from Scientific Polymer Products (Ontario, NY, USA). The average molecular weights $\left(M_{\mathrm{n}}\right.$ and $\left.M_{\mathrm{w}}\right)$ were determined by size exclusion chromatography. The values of $m$ reported in the literature tend to become scattered over wide ranges even for the same polymer material. For example, the reported values for atactic PS range from 80 to $180 .^{11,12}$ It has also been reported that $m$ is quite sensitive to the evaluation method and, in some cases, to impurity (such as residual solvents) and to molecular weight. ${ }^{15,16,28}$ Regarding this point, we exercised additional caution: polymers were dissolved in benzene (1.0 wt\%) and freezedried for $170 \mathrm{~h}$ to remove volatile impurities before use. Furthermore, for poly (ethyl methacrylate) (PEMA), poly(phenyl methacrylate) (PPhMA), poly (benzyl methacrylate) (PBzMA) and poly(cyclohexyl methacrylate) (PCHMA), purification by reprecipitation (three times, from tetrahydrofuran $/ n$-hexane) was needed before the freeze-drying process to obtain reliable results. For stereoregular polymers (isotactic PS, syndiotactic PS, isotactic poly(methyl methacrylate) (PMMA), and syndiotactic PMMA), crystallinity may affect the segmental dynamics in the amorphous phase. To exclude such a crystallinity effect, the polymers were first heated to above their apparent melting temperature for $3 \mathrm{~min}$, followed by quenching to room temperature to prevent crystallization. We confirmed that no signs of crystallinity were detected in a subsequent DSC heating scan.

DSC measurements were performed using a Perkin Elmer (Waltham, MA, USA) Pyris Diamond calorimeter (power-compensation type) equipped with a Perkin Elmer Intra-cooler P2 cooling system. The temperature and heat flow were calibrated with an indium standard, and the measurements were performed in a nitrogen atmosphere. We used standard aluminum pans (Perkin Elmer 0219-0041) that were heated at $550{ }^{\circ} \mathrm{C}$ for $5 \mathrm{~min}$ just before use to remove any contaminants. Typical sample size ranged from 4 to $7 \mathrm{mg}$. Upon collecting DSC data for each sample, we first performed a scan on the above
Table 1 Parameters of polymers used in this study

\begin{tabular}{lcccccl}
\hline Polymer & $M_{n} / k D a$ & $M_{w} / k D a$ & $T_{g} / K$ & $\mu d^{\mathrm{a}}$ & $V_{m} / \mathrm{nm}^{3}$ & $\rho / g \mathrm{~cm}^{-3}$ \\
\hline a-PS & $425^{\mathrm{b}}$ & $451^{\mathrm{b}}$ & 379 & 0.59 & 0.165 & 1.05 \\
i-PS & & $400^{\mathrm{b}}$ & 365 & 0.59 & 0.165 & 1.05 \\
s-PS & & $300^{\mathrm{b}}$ & 370 & 0.59 & 0.165 & 1.05 \\
PMS & 30.4 & 103 & 384 & 0.14 & 0.188 & 1.04 \\
PMOS & 59.0 & 127 & 382 & 1.23 & 0.221 & 1.009 \\
PtBS & 43.6 & 274 & 413 & 0.36 & 0.280 & 0.95 \\
PCIS & 39.6 & 79.3 & 404 & 2.2 & 0.149 & 1.55 \\
PBrS & 33.1 & 61.1 & 418 & 1.9 & 0.216 & 1.408 \\
a-PMMA & $292^{\mathrm{b}}$ & $298^{\mathrm{b}}$ & 405 & 1.72 & 0.138 & 1.2 \\
i-PMMA & 44.3 & 159 & 327 & 1.72 & 0.136 & 1.22 \\
s-PMMA & 42.9 & 56.6 & 401 & 1.72 & 0.140 & 1.19 \\
PEMA & 90.4 & 222 & 349 & 1.78 & 0.172 & 1.1 \\
PiPMA & 50.2 & 110 & 365 & 1.75 & 0.206 & 1.033 \\
PnBMA & 69.8 & 165 & 303 & 1.87 & 0.220 & 1.07 \\
PsBMA & 33.2 & 90.4 & 338 & 1.87 & 0.224 & 1.052 \\
PPhMA & 25.9 & 71.9 & 402 & 1.53 & 0.222 & 1.21 \\
PBzMA & 35.9 & 124 & 335 & 1.22 & 0.248 & 1.179 \\
PCHMA & 26.5 & 95.0 & 380 & 1.92 & 0.254 & 1.10
\end{tabular}

Abbreviations: a-PMMA, atactic poly(methyl methacrylate); a-PS, atactic polystyrene; i-PMMA, isotactic poly(methyl methacrylate); i-PS, isotactic polystyrene; PBrS, poly(4-bromostyrene); PBzMA, poly(benzyl methacrylate); PCHMA, poly(cyclohexyl methacrylate); PCIS, poly(4chlorostyrene); PEMA, poly(ethyl methacrylate); PiPMA, poly(isopropyl methacrylate); PMOS, poly(4-methoxystyrene); PMS, poly(4-methylstyrene); PnBMA, poly(n-butyl methacrylate); PPhMA, poly(phenyl methacrylate); PsBMA, poly(sec-butyl methacrylate); PtBS, poly(4-tertbutylstyrene); s-PMMA, syndiotactic poly(methyl methacrylate); s-PS, syndiotactic polystyrene. The polymers are atactic unless specified.

The polymers are atactic unless specified.

aData from Rosamonte's Physical Chem
bData from manufacturer's data sheet.

purified empty pan, and then we placed the specimen in the same pan and repeated the scan. The obtained trace was subtracted by that of the empty pan. This procedure greatly improved the accuracy and reproducibility of the data because the baseline of traces from the calorimeter of the power-compensation type often became unstable. To check the reproducibility of the data, iterative measurements for different specimens were performed several times for each material.

Fragility parameter $m$ was evaluated based on the cooling rate dependence of fictive temperature $T_{\mathrm{f}}$, based on the assumption that the Frenkel-KobekoReiner constant is independent of the scanning rate. ${ }^{25}$ The polymer sample was first heated to $T_{\mathrm{g}}+50 \mathrm{~K}$ for $2 \mathrm{~min}$, and was then cooled to $T_{\mathrm{g}}-50 \mathrm{~K}$ at cooling rate $q$ that ranged from 1.0 to $20 \mathrm{~K} \mathrm{~min}^{-1}$. Finally, the sample was heated to $T_{\mathrm{g}}+50 \mathrm{~K}$ at $20 \mathrm{~K} \mathrm{~min}^{-1}$, and the obtained heating trace was analyzed to evaluate $T_{\mathrm{f}}{ }^{16,29}$ Here we define $T_{\mathrm{s}}$ as a standard fictive temperature that was observed at a cooling rate $q_{\mathrm{s}}=20.0 \mathrm{~K} \mathrm{~min}^{-1}$. From the slope of $\log \left(q_{\mathrm{s}} / q\right)$ vs $T_{\mathrm{s}} / T_{\mathrm{f}}$ plot, we evaluated $m$. Figure 1 shows a typical example. In this paper, we also used the values of $T_{\mathrm{s}}$ as the (standard) glass transition temperature $T_{\mathrm{g}}$ for convenience, as listed in Table 1.

Cooperative length scale $\xi$ at $T_{\mathrm{g}}$ can be estimated by the temperaturemodulated DSC technique that provides complex heat capacity. In this study, we used an alternative simple method proposed by Donth et al. ${ }^{17,30}$ The feasibility of this method is discussed elsewhere. ${ }^{17,31}$ We performed step-scan heating measurements, a kind of temperature-modulated DSC: ${ }^{32}$ stepwise heating was executed, each step consisting of a heating period up to $2.0 \mathrm{~K}$ at a rate of $5.0 \mathrm{~K} \mathrm{~min}^{-1}$ and an isothermal period with a duration of $<90 \mathrm{~s}$. The temperature range of the step scan was from $T_{\mathrm{g}}-50 \mathrm{~K}$ to $T_{\mathrm{g}}+50 \mathrm{~K}$. From the obtained reversing heat capacity traces, we evaluated the breadth of glass transition $\Delta T$, defined as a temperature difference between the onset and endset of the transition. The temperature fluctuation parameter $\delta T$ was estimated by using a relation $\delta T=\Delta T / C$, where $C$ is a constant. It should be noted that the breadth of glass transition on temperature scanning depends on the scanning rate. ${ }^{25}$ The mean heating rate of the step-scan was $\sim 2.3 \mathrm{~K} \mathrm{~min}^{-1}$ and was almost invariant for each scan, and we used an empirical value that $C=5.0$. We 
confirmed that the obtained $\delta T$ values for PMAEs were consistent with values in the literature. ${ }^{17}$

To evaluate the cooperative length scale $\xi\left(T_{\mathrm{g}}\right)$, we used the following relation based on the fluctuation-dissipation theorem: ${ }^{17}$

$$
\xi^{3}\left(T_{\mathrm{g}}\right)=\frac{k T^{2} \Delta\left(1 / C_{V}\right)}{\rho(\delta T)^{2}}
$$

with

$$
\Delta\left(1 / C_{V}\right)=\frac{1}{C_{V}(\mathrm{~g})}-\frac{1}{C_{V}(l)} \approx \frac{1}{C_{p}(\mathrm{~g})}-\frac{1}{C_{p}(l)}
$$

where $\rho$ is the mass density, $k$ is the Boltzmann constant and $C_{V}(\mathrm{~g})$ and $C_{V}(\mathrm{l})$ are the isochoric heat capacities at $T_{\mathrm{g}}$ in the glassy and liquid states, respectively. Isobaric heat capacities $C_{p}(\mathrm{~g})$ and $C_{p}(\mathrm{l})$ at $T_{\mathrm{g}}$ were evaluated from the reversing heat capacity trace that was obtained by the step-scan measurement. Before the evaluation of the absolute reversing heat capacity, we performed a calibration with respect to a sapphire standard. The obtained values were further checked by comparing them with reported values if available. ${ }^{33} N_{\mathrm{CRR}}\left(T_{\mathrm{g}}\right)$ was evaluated by

$$
N_{\text {CRR }}\left(T_{\mathrm{g}}\right)=\xi^{d}\left(T_{\mathrm{g}}\right) \rho_{n}
$$

where $\rho_{n}$ is the number density of segments, and we assumed $d=3$.

Wide-angle X-ray scattering (WAXS) measurements were performed using a Bruker (Billerica, MA, USA) AXS D8 Advance diffractometer with a $\mathrm{Cu} \mathrm{K \alpha}$ generator operated at $40 \mathrm{kV}$ and $40 \mathrm{~mA}$. For measurements, polymer samples were melt pressed into a mold (trench) with a depth of $0.6 \mathrm{~mm}$ on a glass substrate. Line collimation was used, and $2 \theta / \theta$ scan was performed through the use of a reflection mode. The raw scattering data were corrected for background scattering.

\section{RESULTS}

We found that for the present PS derivatives, $T_{\mathrm{g}}$ becomes high as the bulkiness of the substituent increases (Table 1). $T_{\mathrm{g}}$ is the temperature at which the segmental relaxation time is comparable to the time constant of observation (typically $10^{2}-10^{3} \mathrm{~s}$ ), and may be mainly governed by the main chain stiffness and cohesive energy between segments. ${ }^{34}$ In the PS derivatives, the substituent is linked to the backbone chain via a relatively rigid phenylene unit, so that the substituent directly affects the mobility of the backbone chain dynamics. In contrast to the PS derivatives, $T_{\mathrm{g}}$ of PMAEs exhibits no clear trend with respect to the bulkiness of the ester (Table 1): the ester group is linked via a flexible -COO- linkage that makes the

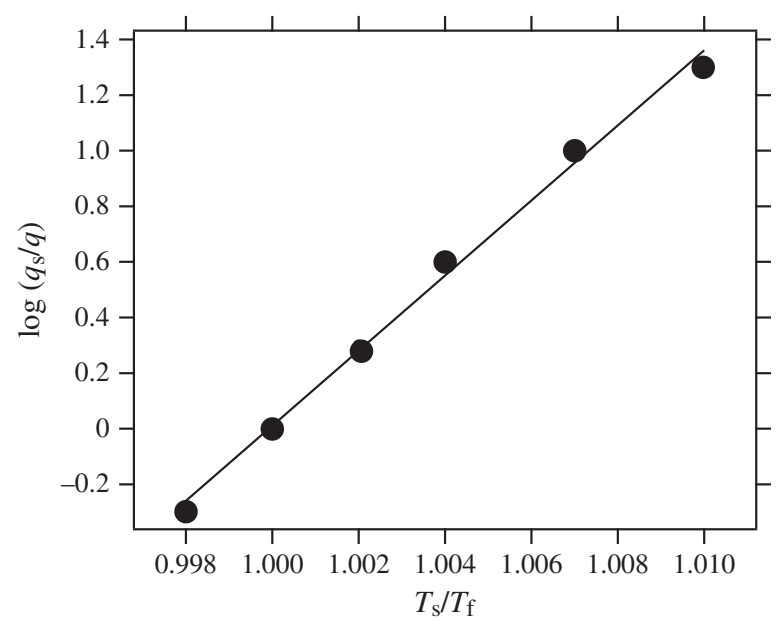

Figure 1 Typical plot of $\log \left(q_{\mathrm{s}} / q\right)$ vs $T_{\mathrm{s}} / T_{\mathrm{f}}$ for polystyrene. The solid line was obtained by linear regression analysis, and the fragility parameter $m$ was evaluated from its slope. substituent effect indirect. Librational motion of phenyl group has been found to occur in polystyrene even at $200 \mathrm{~K}$ (far below $T_{\mathrm{g}}$ ), ${ }^{35}$ suggesting that the phenylene units in the present PS derivatives have some degree of deformability around $T_{\mathrm{g}}$. Nevertheless, phenylene unit is considered to be relatively rigid compared with the ester linkage in PMAEs.

The relation between $m$ and $T_{\mathrm{g}}$ has been investigated for a wide variety of glass formers, and a positive correlation has been found regarding polymeric materials. ${ }^{36}$ This might be simply understood as meaning that less mobile (higher $T_{\mathrm{g}}$ ) materials have greater activation energy. Indeed, it is derived from the Williams-Landel-Ferry relation that $m$ is proportional to $T_{\mathrm{g}}$ and the apparent activation energy to $T_{\mathrm{g}}{ }^{2} \cdot{ }^{37}$ However, Agapov et al. ${ }^{37}$ reported that $T_{\mathrm{g}}$ is raised whereas $m$ decreases when intermolecular interactions become stronger as in the
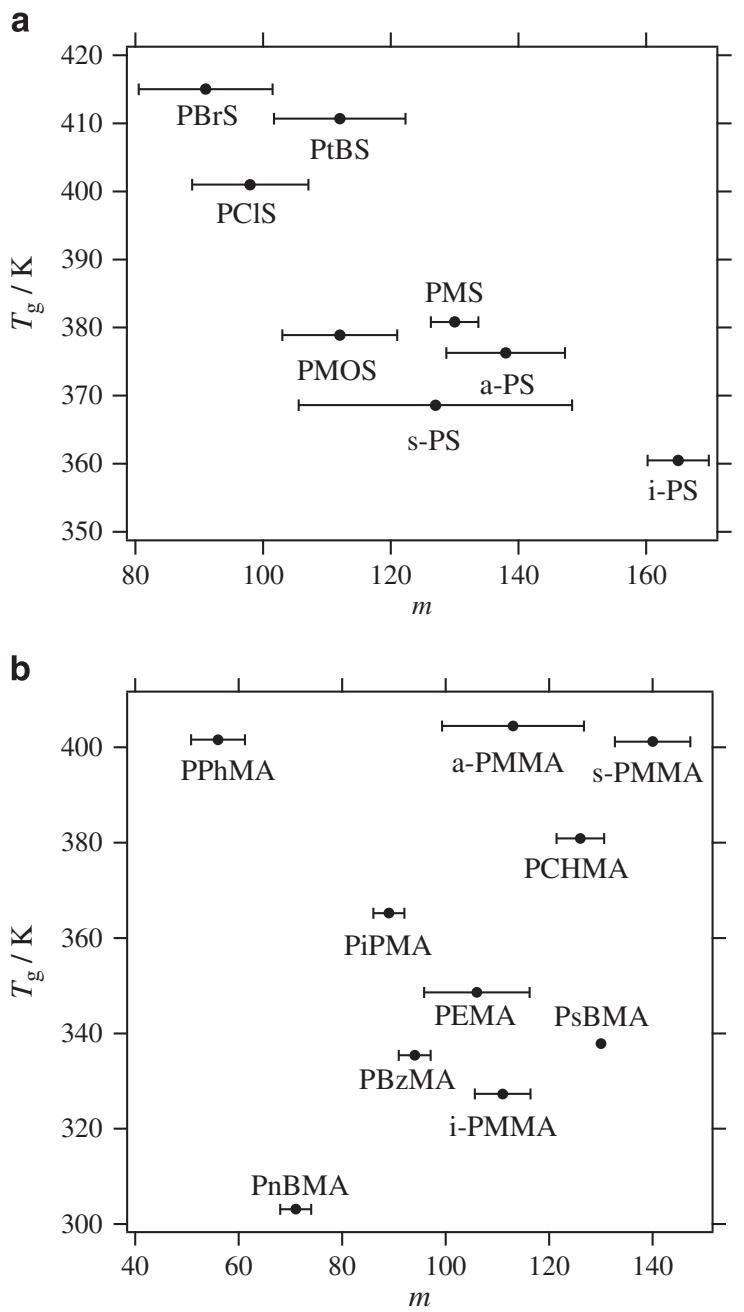

Figure 2 Glass transition temperature $T_{\mathrm{g}}$ plotted against fragility $m$ for (a) polystyrene derivatives and (b) poly(methacrylic acid ester)s. a-PMMA, atactic poly(methyl methacrylate); a-PS, atactic polystyrene; i-PMMA, isotactic poly(methyl methacrylate); i-PS, isotactic polystyrene; PBrS, poly(4bromostyrene); PBzMA, poly(benzyl methacrylate); PCHMA, poly(cyclohexyl methacrylate); PCIS, poly(4-chlorostyrene); PEMA, poly(ethyl methacrylate); PiPMA, poly(isopropyl methacrylate); PMOS, poly(4-methoxystyrene); PMS, poly(4-methylstyrene); PnBMA, poly(n-butyl methacrylate); PPhMA, poly (phenyl methacrylate); PsBMA, poly(sec-butyl methacrylate); PtBS, poly(4tert-butylstyrene); s-PMMA, syndiotactic poly(methyl methacrylate); s-PS, syndiotactic polystyrene. 
case of poly(4-methylstyrene) and poly(4-chlorostyrene). Figure 2 shows a negative correlation for PS derivatives. This result implies that $m$ tends to decrease as the bulkiness of the substituent increases. This may be understood by considering that cooperativity decreases as the bulkiness of the substituent increases. This point will be discussed later.

On the other hand, just a very weak positive correlation is observed for PMAEs if we neglect the point for PPhMA. Such a correlation of PMAEs opposite to that of the PS derivatives seems to be interesting, but no systematic tendency with respect to the bulkiness of the ester part is found. In addition, the correlation is weaker than that for the PS derivatives: linear correlation coefficient $r$ was estimated to be 0.622 if the data point of PPhMA is excluded, whereas $r=-0.849$ for the PS derivatives. These results suggest that variation of the ester part leads to different effects on the dynamics with respect to their chemical nature. Regarding this point, we infer that the ester part in PMAE can contact with various part of the polymer molecule (backbone and side group) because of the flexible -COO- linkage. Here we solely emphasize that the PS system exhibits rather simple and systematic trends for both $T_{\mathrm{g}}$ and $m$ with respect to the substituent.

For PS derivatives, a distinct correlation between $N_{\mathrm{CRR}}\left(T_{\mathrm{g}}\right)$ and $m$ was observed (Figure 3), whereas no apparent correlation was discernible for PMAEs. Linear correlation coefficient $r$ was found to be 0.863 and 0.126 for the PS derivatives and PMAEs (neglecting PPhMA), respectively. A similar result was again observed in the plots of $\xi^{3}\left(T_{\mathrm{g}}\right)$ vs $m$ as shown in Figure 4 . The results suggest that for PS derivatives, the effects of the substituent on $T_{\mathrm{g}}, m$ and the cooperativity parameters vary consistently, whereas for PMAEs the side group may affect the backbone chain dynamics in a rather complex manner, of which interpretation requires a more detailed consideration of the chemical structure. However, rather clear correlation is seen if the data points of PPhMA, isotactic PMMA and PBzMA are excluded. The open squares in Figure $3 \mathrm{~b}$ are the literature values from Hemmpel et $a l .{ }^{17}$ for PMAEs with $n$-alkyl esters. These data seems to be consistent with the present result, suggesting a positive correlation between cooperativity and fragility. The length of the linear alkyl group may cause the segmental dynamics to vary systematically. ${ }^{27}$ The other types of ester part such as benzyl and phenyl groups may affect the backbone dynamics in an appreciably different way from the linear alkyl groups, so that their data points are located far off the correlation line of the $\operatorname{poly}(n$-alkyl methacrylate)s.

The effects of stereoregularity on $T_{\mathrm{g}}, m$ and cooperativity were revealed to be insignificant for PS, though isotactic PS exhibits slightly greater fragility. On the other hand, isotactic PMMA exhibits a remarkably lower $T_{\mathrm{g}}$ and higher cooperativity than the other two PMMAs. The low $T_{\mathrm{g}}$ of isotactic PMMA is partially because of its low backbone stiffness as evidenced by the static stiffness parameter. ${ }^{38}$ It has been revealed that dynamic stiffness (segmental relaxation time) increases generally as static chain stiffness increases in dilute solutions. ${ }^{39,40}$

Figure 5 shows the WAXS profiles for the present PS derivatives. It is known that polystyrene exhibits two dominant halos at around $s=7.1$ and $\left.13.5 \mathrm{~nm}^{-1}(s=(4 \pi / \lambda) \sin \theta)\right)^{41-43}$ The lower $s$ peak is called the polymerization peak because of its absence in the styrene monomer. ${ }^{41}$ In Figure 5, the lower $s$ peak is clearly observed for atactic PS and poly(4-methylstyrene), whereas for the other PS derivatives the lower $s$ peak is very weak. Table 3 shows locations and intensity ratio of the two major peaks: $I_{1}$ and $I_{2}$ are the peak intensities at $s_{1}$ and $s_{2}$, respectively. Here, the peak intensity was evaluated from an experimental polynomial for the peak region obtained by the nonlinear least squares fitting method.
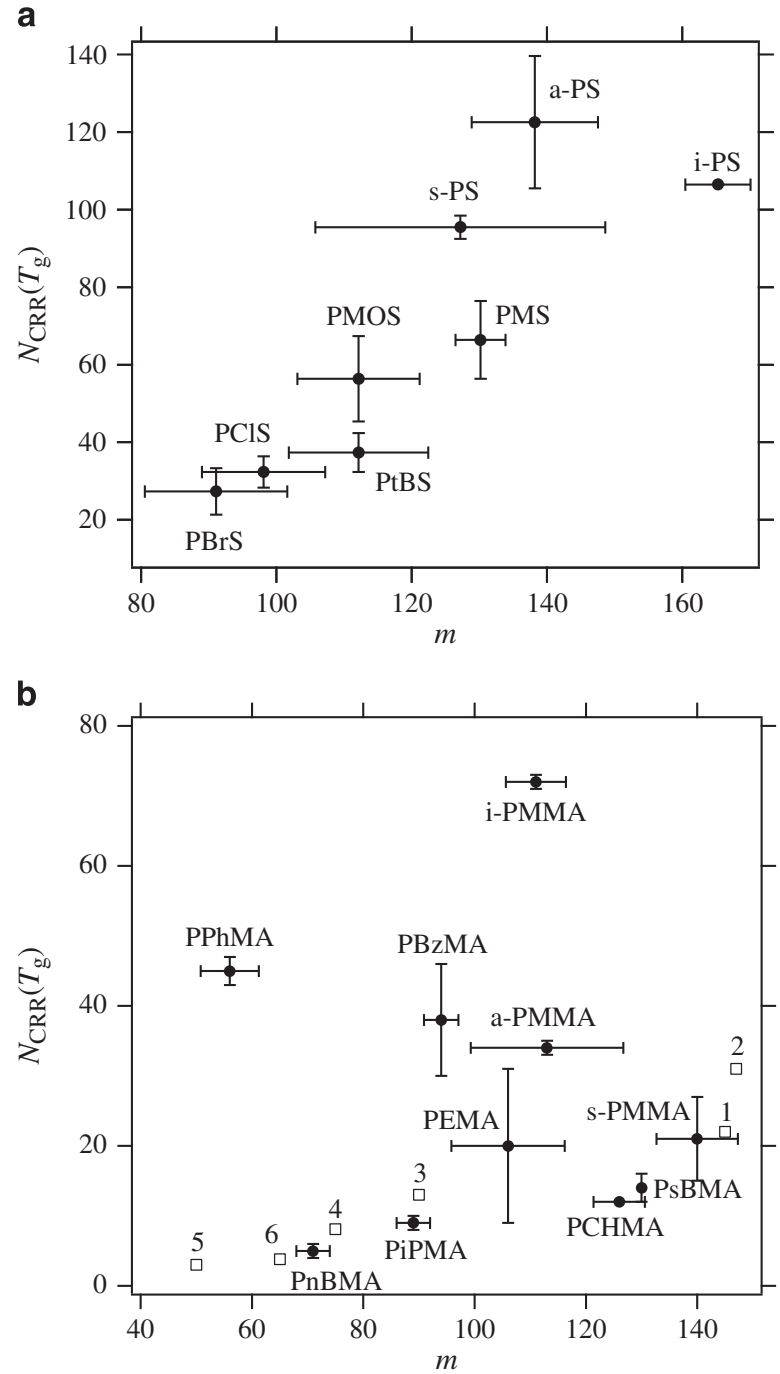

Figure 3 Cooperativity parameter $N_{\mathrm{CRR}}\left(T_{\mathrm{g}}\right)$ plotted against fragility $m$ for (a) polystyrene derivatives and (b) poly(methacrylic acid ester)s. The open squares represent data on poly(n-alkyl methacrylate)s from Hemmpel et al ${ }^{17}$ with alkyl group of 1: methyl, 2: ethyl, 3: propyl, 4: butyl, 5: pentyl and 6: hexyl. a-PMMA, atactic poly(methyl methacrylate); a-PS, atactic polystyrene; CRR, cooperatively rearranging region; i-PMMA, isotactic poly(methyl methacrylate); i-PS, isotactic polystyrene; PBrS, poly(4-bromostyrene); PBzMA, poly(benzyl methacrylate); PCHMA, poly(cyclohexyl methacrylate); PCIS, poly(4-chlorostyrene); PEMA, poly(ethyl methacrylate); PiPMA, poly (isopropyl methacrylate); PMOS, poly(4-methoxystyrene); PMS, poly(4methylstyrene); PnBMA, poly( $n$-butyl methacrylate); PPhMA, poly(phenyl methacrylate); PsBMA, poly(sec-butyl methacrylate); PtBS, poly(4-tertbutylstyrene); s-PMMA, syndiotactic poly(methyl methacrylate); s-PS, syndiotactic polystyrene.

\section{DISCUSSION}

\section{Correlation between cooperativity and fragility}

According to the Adam-Gibbs theory, ${ }^{5}$ the segmental relaxation time $\tau(T)$ depends on both temperature and configurational entropy as

$$
\tau(T)=\tau_{0} \exp \left(\frac{\Delta \mu s_{\mathrm{c}}^{*}}{k T S_{\mathrm{c}}(T)}\right)
$$

where $S_{\mathrm{c}}$ is the configurational entropy per segment, $s_{\mathrm{c}}{ }^{*}$ the configurational entropy of the smallest CRR and $\Delta \mu$ the activation 


\section{a}

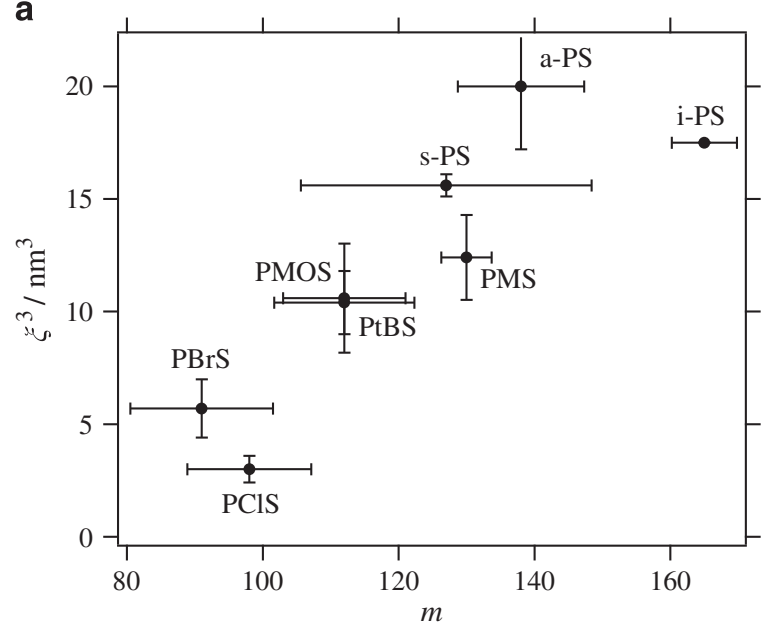

b

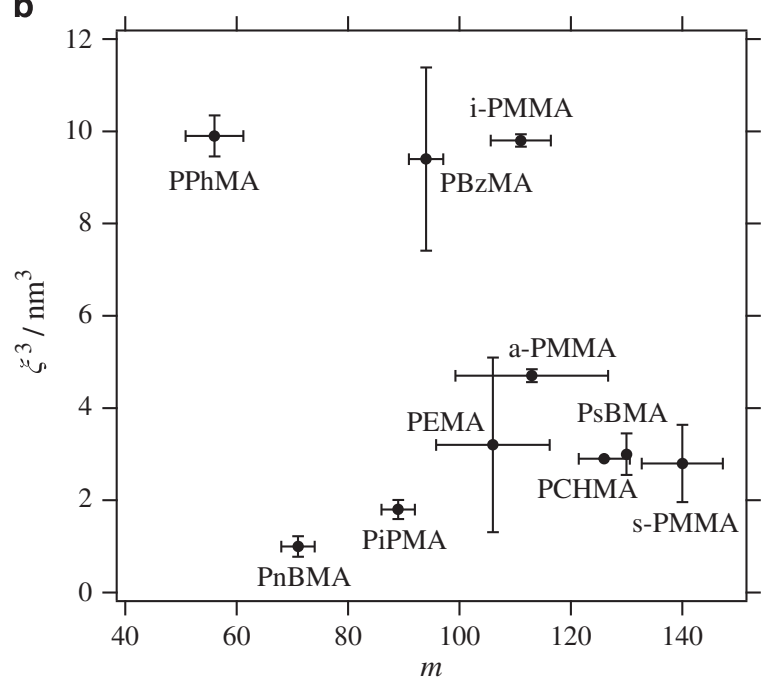

Figure 4 Cooperatively rearranging volume $\xi^{3}\left(T_{\mathrm{g}}\right)$ plotted against fragility $m$ for (a) polystyrene derivatives and (b) poly(methacrylic acid ester)s. a-PMMA, atactic poly(methyl methacrylate); a-PS, atactic polystyrene; i-PMMA, isotactic poly(methyl methacrylate); i-PS, isotactic polystyrene; PBrS, poly(4bromostyrene); PBzMA, poly(benzyl methacrylate); PCHMA, poly(cyclohexyl methacrylate); PCIS, poly(4-chlorostyrene); PEMA, poly(ethyl methacrylate); PiPMA, poly(isopropyl methacrylate); PMOS, poly(4-methoxystyrene); PMS, poly(4-methylstyrene); PnBMA, poly( $n$-butyl methacrylate); PPhMA, poly (phenyl methacrylate); PsBMA, poly(sec-butyl methacrylate); PtBS, poly(4tert-butylstyrene); s-PMMA, syndiotactic poly(methyl methacrylate); s-PS, syndiotactic polystyrene.

energy per segment. From the definition of $m$, we obtain

$$
m=\frac{N_{\mathrm{CRR}}\left(T_{\mathrm{g}}\right) \Delta \mu \log e}{k T_{\mathrm{g}}}
$$

A positive correlation is expected from Equation (6) if $\Delta \mu / T_{\mathrm{g}}$ does not depend on the material to a great degree, and this seems to be the case for the PS derivatives. This is reasonably understood by the higher barrier that has to be overcome for a larger (bulkier) substituent that simultaneously reduces the segmental mobility ( $T_{\mathrm{g}}$ is raised), that is, both $\Delta \mu$ and $T_{\mathrm{g}}$ increase with an increase in the bulkiness of substituent. We estimated $\Delta \mu$ using Equation (6) as shown in Table 2. Calculation based on lattice cluster theory suggests that for

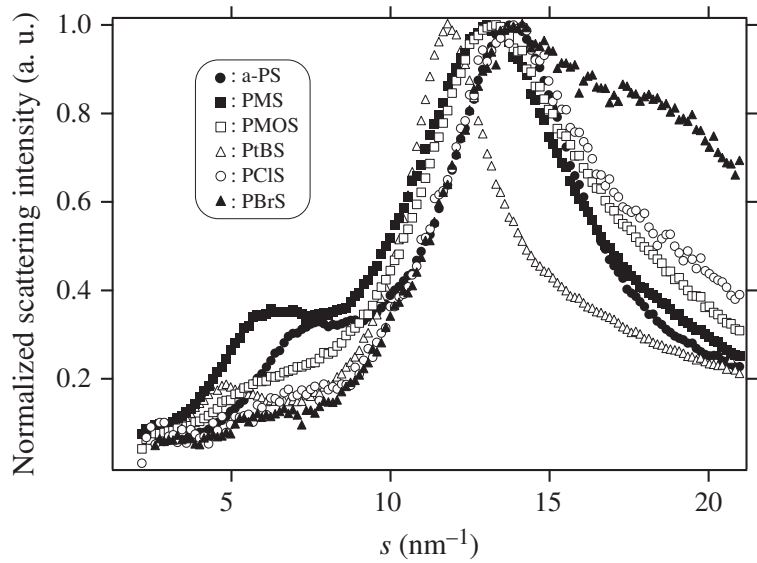

Figure 5 Wide-angle X-ray scattering profiles for the polystyrene (PS) derivatives. The scattering intensity is normalized with respect to the maximum peak height. a-PS, atactic polystyrene; PBrS, poly(4bromostyrene); PCIS, poly(4-chlorostyrene); PMOS, poly(4-methoxystyrene); PMS, poly(4-methylstyrene); PtBS, poly(4-tert-butylstyrene).

Table 2 Activation energy per segment estimated from Equation (6)

\begin{tabular}{lcc}
\hline Polymer & $\Delta \mu / 10^{-20} \mathrm{~J}$ & $\Delta \mu / \mathrm{k} / 10^{3} \mathrm{~K}$ \\
\hline a-PS & 1.36 & 0.99 \\
i-PS & 1.80 & 1.31 \\
s-PS & 1.57 & 1.14 \\
PMS & 2.41 & 1.74 \\
PMOS & 2.43 & 1.76 \\
PtBS & 3.93 & 2.88 \\
PCIS & 3.93 & 2.85 \\
PBrS & 4.48 & 3.23 \\
\hline
\end{tabular}

Abbreviations: a-PS, atactic polystyrene; i-PS, isotactic polystyrene; PBrS, poly(4-bromostyrene); PCIS, poly(4-chlorostyrene); PMOS, poly(4-methoxystyrene); PMS, poly(4-methylstyrene); PtBS, poly(4-tert-butylstyrene); s-PS, syndiotactic polystyrene.

a polymer with a flexible backbone, the stiffness of the side group raises $\Delta \mu / k,{ }^{44,45}$ consistent with the results shown in Table 2. Here, the value of $\Delta \mu$ varies quite reasonably with respect to the substituent bulkiness. An empirical relation $\Delta \mu / k=a T_{\mathrm{mc}}$ has been proposed, where $T_{\mathrm{mc}}$ is the experimental crossover temperature of the mode coupling theory ${ }^{46-48}$ with $a=6-7.44,49$ If $T_{\mathrm{mc}}$ is largely proportional to $T_{\mathrm{g}}$ as predicted by the mode-coupling theory $\left(T_{\mathrm{mc}}=1.2 T_{\mathrm{g}}\right)$, constancy of $\Delta \mu / T_{\mathrm{g}}$ is derived.

\section{Structures in PS derivatives}

As pointed above, the cooperativity parameter $N_{\mathrm{CRR}}\left(T_{\mathrm{g}}\right)$ for the PS derivatives decreases as the size of substituent becomes larger, implying that cooperative feature of segmental relaxation becomes prominent for the smallest substituent $(-\mathrm{H})$, that is, polystyrene. We infer that this is relevant to the packing structure of the chains in an amorphous state. The lower $s$ peak in the WAXS profile mainly reflects backbone-backbone correlation that has been assumed to be related to stacking of phenyl groups resulting in a superchain structure. ${ }^{43}$ The higher $s$ peak, on the other hand, is the most intense one and mainly reflects phenyl-phenyl correlation. The existence of the polymerization peak suggests certain additional structural order in PS that may 
Table 3 Parameters estimated from WAXS profile

\begin{tabular}{lcccc}
\hline Polymer & $s_{1} / n m^{-1}$ & $s_{2} / n m^{-1}$ & $I_{1} / I_{2}$ & $\xi\left(T_{g}\right) s_{2} / 2 \pi$ \\
\hline a-PS & 7.73 & 13.8 & 0.325 & 6.0 \\
PMS & 6.44 & 13.1 & 0.358 & 4.8 \\
PMOS & 5.53 & 13.3 & 0.183 & 4.6 \\
PtBS & 4.81 & 11.8 & 0.207 & 4.1 \\
PCIS & 6.3 & 13.8 & 0.144 & 3.2 \\
PBrS & 6.3 & 14.0 & 0.103 & 4.0 \\
\hline
\end{tabular}

Abbreviations: a-PS, atactic polystyrene; PBrS, poly(4-bromostyrene); PCIS, poly(4chlorostyrene); PMOS, poly(4-methoxystyrene); PMS, poly(4-methylstyrene); PtBS, poly(4-tertbutylstyrene).

enhance the cooperativity in segmental rearrangement. One interesting feature is that the lower $s$ peak increases with increasing temperature, whereas the higher $s$ peak slightly decreases with increasing temperature. Interpretation of this behavior is rather complicated. Various types of atomic pairs, for example, backbone-backbone, phenyl-phenyl and backbone-phenyl atomic pairs, and also intra and inter-chain atomic pairs contribute to the two halos. Contribution of each type behaves in quite a different manner with temperature. Detailed discussion on this issue is made on the basis of simulation work. ${ }^{50-53}$ We should also note that the lower $s$ peak is sensitive to the packing structure as evidenced by the physical aging experiments. ${ }^{54}$

The result in Table 3 shows that $I_{1} / I_{2}$ becomes smaller as the substituent becomes bulkier. It is likely that the para-substituent weakens backbone-backbone correlation, leading to a reduction in segmental cooperativity. Bulky para-substituents probably prevent the stacking of phenyl groups that, as a result, reduces the strength of backbone-backbone correlation.

The value of $s_{1}$ tends to decrease with an increase in the substituent's bulkiness (Table 3). This may be explained by an increase in the correlation distance between backbone chains when the substituent becomes larger. On the other hand, $s_{2}$ is almost constant except for poly(4-tert-butylstyrene) (PtBS). This constancy may indicate that the phenyl-phenyl correlation is not perturbed significantly by the substituent even for - Br group. For PtBS, tert-butyl group occupies a large space than $-\mathrm{Cl}$ or $-\mathrm{Br}$, and hence it may appreciably hinder the stacking structure of phenyl groups. The ratio of $\xi\left(T_{\mathrm{g}}\right)$ to phenyl-phenyl distance is estimated by $\xi\left(T_{\mathrm{g}}\right) s_{2} / 2 \pi$, as listed in Table 3 . The obtained values lie within the range of various theoretical predictions of 3-6. ${ }^{7,10,20,55}$ The tendency of substituent dependence of $\xi\left(T_{\mathrm{g}}\right) s_{2} / 2 \pi$ seems to be a little different from that of $I_{1} / I_{2}$ : poly(4-chlorostyrene) gives the lowest value for the former, whereas poly(4-bromostyrene) does so for the latter. This may reflect a fundamental difference in these parameters, that is, the cooperativity concerns dynamical phenomenon whereas WAXS characterizes static structural features.

\section{Packing efficiency}

It has been argued that fragility depends on the packing efficiency of the segments. ${ }^{44,56}$ It may be intuitively understood that dense packing requires significant additional volume on rearrangement, leading to a high fragility (or a high barrier). Unfortunately, we found no clear correlation for $m$ vs $V_{\mathrm{m}}$, as well as for $m$ vs $\rho$ (mass density) for either the PS or PMAE systems (Table 1). $V_{\mathrm{m}}$ is a measure of segmental size, and $\rho$ depends strongly on the elements included in the polymer. Neither of these may be an appropriate parameter for evaluating the packing efficiency. Recently, the pressure dependence of glass transition dynamics has been extensively investigated. ${ }^{57}$ It has been claimed that the activation volume, which practically determines the contribution of volume to fragility, should be correlated with cooperativity. ${ }^{7}$ In addition, a clear positive correlation has been revealed between the activation volume and $\xi\left(T_{\mathrm{g}}\right)$ for polymeric materials that have no similarity in chemical structure. ${ }^{23}$ The correlation between $\xi^{3}\left(T_{\mathrm{g}}\right)$ and $m$ observed for the present PS system suggests a dominant effect of the substituent's bulkiness on the dynamics rather than the cohesive energetic contribution from the para-substituent. We speculate that the isochoric part of the fragility plays merely a minor role in the dynamics of the PS derivatives.

The above consideration may be supported by values of the dipole moment $\mu_{0}$ of the monomer model compound shown in Table $1 .{ }^{58}$ Here $\mu_{0}$ may be a measure of nonbonded interactions between segments that are directly related to the cohesive energy between segments. If we assume that the degree of cooperativity in segmental relaxation is largely governed by the cohesive energy, we might expect a certain type of correlation to exist between $N_{\mathrm{CRR}}\left(T_{\mathrm{g}}\right)$ and $\mu_{0}$. However, we found no apparent correlation between the two parameters (Table 1). In particular, halogen-containing PS polymers show high $\mu_{0}$ values but low cooperativity. Thus, spatial hindrance due to the substituent is suggested to play a dominant role in the PS derivatives.

The packing efficiency may be related to the free volume in glassy materials. It has been pointed out that, in general, a material with lower fragility has less free volume. ${ }^{59}$ From the present data, the thermal expansion coefficient of the fractional free volume $\alpha_{\mathrm{f}}$ can be estimated by using a relation $\alpha_{\mathrm{f}}=k / \Delta \mu{ }^{59}$ The result for the PS derivatives in Table 2 indicates that $\alpha_{\mathrm{f}}$ becomes smaller as $m$ decreases. This might be consistent with the general trend of the correlation between free volume fraction and $m$.

\section{Configurational entropy}

It has been reported that for a large number of glass formers other than polymers, the configurational entropy of the smallest CRR, $s_{\mathrm{c}}{ }^{*}$ depends on temperature, and a universal curve in the plot of $s_{\mathrm{c}}{ }^{*}$ vs segmental relaxation time has been found. ${ }^{60}$ Although the temperature dependence of $s_{\mathrm{c}}{ }^{*}$ is inconsistent with the assumption of the original Adam-Gibbs model, ${ }^{5}$ random first-order transition theory predicts that $s_{\mathrm{c}}{ }^{*}$ increases with increasing temperature. ${ }^{22}$ The above universality implies that $s_{\mathrm{c}}{ }^{\star}\left(T_{\mathrm{g}}\right)$ is almost invariant with respect to material. The value of $s_{\mathrm{c}}{ }^{\star}\left(T_{\mathrm{g}}\right)$ may be $\sim 5-6 \times 10^{-21} \mathrm{~J} \mathrm{~K}^{-1}$ for nonpolymeric glass formers. ${ }^{60}$ This may not be the case for polymeric materials because of the complex interactions between segments (including bonded and nonbonded interactions). However, from the obvious correlation between $N_{\mathrm{CRR}}\left(T_{\mathrm{g}}\right)$ and $m$ observed for the present PS derivatives, we infer that $s_{\mathrm{c}}{ }^{*}\left(T_{\mathrm{g}}\right)$ does not vary significantly with respect to the substituent. In this case, the configurational entropy per segment $S_{\mathrm{c}}\left(T_{\mathrm{g}}\right) \quad\left(=s_{\mathrm{c}}{ }^{\star} / N_{\mathrm{CRR}}\left(T_{\mathrm{g}}\right)\right)$ is expected to increase as the substituent becomes bulkier. This point will be investigated in a forthcoming paper.

\section{CONCLUSIONS}

We investigated the glass transition temperature, fragility and cooperativity of PS derivatives possessing various para-substituents on the phenyl group. The following results were obtained: (1) glass transition temperature is raised consistently as the bulkiness of the parasubstituent increases, (2) both cooperativity parameters, $N_{\mathrm{CRR}}\left(T_{\mathrm{g}}\right)$ and $\xi^{3}\left(T_{\mathrm{g}}\right)$, exhibit a tendency to increase with increasing fragility and (3) both fragility and cooperativity tend to decrease as the substituent's 
bulkiness increases. WAXS results suggested that the structural order that is responsible for the lower $s$ peak is weakened by the bulkiness of the substituent, leading to the reduction of cooperativity in segmental dynamics. The systematic trends of glass transition dynamics seem to be characteristic to the present PS derivatives, where the substituent is attached to the backbone chain via phenyl ring. In this case, the origin of the substituent's effects on the parameters may be rather simple because of the strong limitation of its spatial arrangement via phenyl group: the substituent plays a major role in changing the packing ability. Contrary to the PS system, PMAEs exhibit rather complex behaviors with less weaker correlations between the parameters. It is likely that the flexible ester linkage allows a variety of spatial arrangements of the substituent, leading to various types of segmental interaction.

\section{ACKNOWLEDGEMENTS}

We thank Aoto Kakinoki and Akinori Sato for their help with the DSC measurements. We thank Professor Kazunori Se for his help with the molecular weight determination.

1 Ediger, M. D., Angell, C. A. \& Nagel, S. R. Supercooled liquids and glasses. J. Phys. Chem. 100, 13200-13212 (1996).

2 Roland, C. M. Relaxation phenomena in vitrifying polymers and molecular liquids. Macromolecules 43, 7875-7890 (2010).

3 Ediger, M. D. \& Harrowell, P. Perspective: Supercooled liquids and glasses. J. Chem. Phys. 137, 080901 (2012)

4 Freed, K. F. Towards first principles theory of relaxation in supercooled liquids formulated in terms of cooperative motion. J. Chem. Phys. 141, 141102 (2014).

5 Adam, G. \& Gibbs, J. H. On the temperature dependence of cooperative relaxation properties in glass-forming liquids. J. Chem. Phys. 43, 139-146 (1965).

6 Casalini, R., Fragiadakis, D. \& Roland, C. M. Dynamic correlation length scales under isochronal conditions. J. Chem. Phys. 142, 064504 (2015).

7 Hong, L., Novikov, V. N. \& Sokolov, A. P. Is there a connection between fragility of glass forming systems and dynamic heterogeneity/cooperativity? J. Non-Cryst. Solids 357, 351-356 (2011).

8 Tracht, U., Wilhelm, M., Heuer, A., Feng, H., Schmidt-Rohr, K. \& Spiess, H. W. Length scale of dynamic heterogeneities at the glass transition determined by multidimensional nuclear magnetic resonance. Phys. Rev. Lett. 81, 2727-2730 (1998)

9 Di, X. \& McKenna, G. Evaluation of heterogeneity measures and their relation to the glass transition. J. Chem. Phys. 138, 12 A530 (2013).

10 Schweizer, K. S. \& Saltzman, E. J. Activated hopping, barrier fluctuations, and heterogeneity in glassy suspensions and liquids. J. Phys. Chem. B 108, 19729-19741 (2004).

11 Böhmer, R., Ngai, K. L., Angell, C. A. \& Plazek, D. J. Nonexponential relaxations in strong and fragile glass formers. J. Chem. Phys. 99, 4201-4209 (1993).

12 Huang, D. \& McKenna, G. B. New insights into the fragility dilemma in liquids. J. Chem. Phys. 114, 5621-5630 (2001).

13 Kunal, K., Robertson, C. G., Pawlus, S., Hahn, S. F. \& Sokolov, A. P. Role of chemical structure in fragility of polymers: a qualitative picture. Macromolecules 41 , 7232-7238 (2008)

$14 \mathrm{Hu}$, Y.-F., Zhang, X.-M. \& Song, M. An approach for prediction of dynamic fragility of polymeric glasses. Macromolecules 43, 7391-7393 (2010).

15 Ellison, C. J., Mundra, M. K. \& Torkelson, J. M. Impacts of polystyrene molecular weight and modification to the repeat unit structure on the glass transition - Nanoconfinement effect and the cooperativity length scale. Macromolecules 38, 1767-1778 (2005).

16 Evans, C. M., Deng, H., Jager, W. F. \& Torkelson, J. M. Fragility is a key parameter in determining the magnitude of $T_{\mathrm{s}}$-confinement effects in polymer films. Macromolecules 46, 6091-6103 (2013).

17 Hemmpel, E., Hensel, A., Schick, C. \& Donth, E. Characteristic length of dynamic glass transition near $T_{\mathrm{g}}$ for a wide assortment of glass-forming substances. J. Phys. Chem. B 104, 2460-2466 (2000).

18 Casalini, R. \& Roland, C. M. Effect of regioisomerism on the local dynamics of polychlorostyrene. Macromolecules 47, 4087-4093 (2014)

19 Huth, H., Beiner, M. \& Donth, E. Temperature dependence of glass-transition cooperativity from heat-capacity spectroscopy: two post-Adam-Gibbs variants. Phys. Rev. B 61, 15092-15101 (2000).

20 Dalle-Ferrier, C., Thibierge, C., Alba-Simionesco, C., Berthier, L., Biroli, G., Bouchaud, J.-P., Ladieu, F., L'Hôte, D. \& Tarjus, G. Spatial correlations in the dynamics of glassforming liquids: experimental determination of their temperature dependence. Phys. Rev. E 76, 041510 (2007).

21 Tracht, U., Wilhelm, M., Heuer, A. \& Spiess, H. W. Combined reduced 4D ${ }^{13} \mathrm{C}$ exchange and ${ }^{1} \mathrm{H}$ spin diffusion experiment for determining the length scale of dynamic heterogeneities. J. Magn. Reson. 140, 460-470 (1999).
22 Lubchenko, V. \& Wolynes, P. G. Theory of structural glasses and supercooled liquids. Ann. Rev. Phys. Chem. 58, 235-266 (2007).

23 Hong, L., Gujrati, P. D., Novikov, V. N. \& Sokolov, A. P. Molecular cooperativity in the dynamics of glass-forming systems: a new insight. J. Chem. Phys. 131, 194511 (2009).

24 Sasaki, T., Uchida, T. \& Sakurai, K. Effect of crosslink on the characteristic length of glass transition of network polymers. J. Polym. Sci. B Polym. Phys. 44, 1958-1966 (2006).

25 Schawe, J. E. K. Vitrification in a wide cooling rate range: the relations between cooling rate, relaxation time, transition width, and fragility. J. Chem. Phys. 141, 184905 (2014).

26 Chua, Y. Z., Schulz, G., Shoifet, E., Huth, H., Zorn, R., Scmelzer, J. W. P. \& Schick, C. Glass transition cooperativity from broad band heat capacity spectroscopy. Colloid Polym. Sci. 292, 1893-1904 (2014).

27 Hempel, E., Huth, H. \& Beiner, M. Interrelation between side chain crystallization and dynamic glass transitions in higher poly(n-alkyl methacrylates). Thermochim. Acta 403 , 105-114 (2003)

28 Elfadl, A. A., Herrmann, A., Hintermeyer, J., Petzold, N., Novikov, V. N. \& Rössler, E. A. Molecular weight dependence of fragility in polymers. Macromolecules 42 6816-6817 (2009).

29 Moynihan, C. T., Easteal, A. J., DeBolt, M. A. \& Tucker, J. Dependence of the fictive temperature of glass on cooling rate. J. Am. Ceram. Soc. 59, 12-16 (1976).

30 Donth, E. Characteristic length of the glass transition. J. Polym. Sci. B Polym. Phys. 34, 2881-2892 (1996).

31 Donth, E., Korus, J., Hempel, E. \& Beiner, M. Comparison of DSC heating rate and HCS frequency at the glass transition. Thermochim. Acta 304/305, 239-249 (1997).

32 Sasaki, T., Yamauchi, N., Irie, S. \& Sakurai, K. Differential scanning calorimetry study on thermal behaviors of freeze-dried poly(L-lactide) from dilute solutions. J. Polym. Sci. B Polym. Phys. 43, 115-124 (2005).

33 Pyda M.(ed)ATHAS Data Bank http://materials.springer.com/.

$34 \mathrm{Xu}$, W.-S. \& Freed, K. F. Influence of cohesive energy and chain stiffness on polymer glass formation. Macromolecules 47, 6990-6997 (2014).

35 Kanaya, T., Kawaguchi, T. \& Kaji, K. Fast process of amorphous polystyrene below and above the glass transition temperature $T_{\mathrm{g}}$ as studied by quasielastic neutron scattering. J. Chem. Phys. 104, 3841-3850 (1996).

36 Qin, Q. \& McKenna, G. B. Correlation between dynamic fragility and glass transition temperature for different classes of glass forming liquids. J. Non-Cryst. Solids 352 , 2977-2985 (2006).

37 Agapov, A. L., Wang, Y., Kunal, K., Robertson, C. G. \& Sokolov, A. P. Effect of polar interactions on polymer dynamics. Macromolecules 45, 8430-8437 (2012).

38 Yamakawa, H. A hypothesis on polymer chain configurations. Helical wormlike chains. Macromolecules 10, 692-696 (1977).

39 Yamakawa, H., Yoshizaki, T. \& Fujii, M. Dynamics of helical worm-like chains. VIII. Higher order subspace approximations to dielectric and magnetic relaxation and fluorescence depolarization for flexible chains. J. Chem. Phys. 84, 4693-4707 (1986).

40 Sasaki, T., Arisawa, H. \& Yamamoto, M. Fluorescence depolarization study on local motions of anthracene-labeled poly(alkyl methacrylate)s in dilute solutions and evaluation of their chain stiffness. Polym. J. 23, 103-115 (1991).

41 Katz, J. R. X-ray spectrography of polymers and in particular those having a rubber-like extensibility. Trans. Faraday Soc. 32, 77-96 (1936).

42 Schubach, H. R., Nagy, E. \& Heise, B. Short range order of amorphous polymers derived by WAXS. Colloid Polym. Sci. 259, 789-796 (1981).

43 Mitchell, G. R. \& Windle, A. H. Structure of polystyrene glasses. Polymer 25 , 906-920 (1984).

44 Dudowicz, J., Freed, K. F. \& Douglas, J. F. Fragility of glass-forming polymer liquids. J. Phys. Chem. B 109, 21350-21356 (2005).

45 Dudowicz, J., Freed, K. F. \& Douglas, J. F. Generalized entropy theory of polymer glass formation. Adv. Chem. Phys. 137, 125-222 (2008).

46 Bengtzelius, U., Götze, W. \& Sjölander, A. Dynamics of supercooled liquids and the glass transition. J. Phys. C 17, 5915-5934 (1984).

47 Leutheusser, E. Dynamical model for the liquid-glass transition. Phys. Rev. A 29, 2765-2773 (1984).

48 Götze, W. \& Sjögren, L. Relaxation processes in supercooled liquids. Rep. Prog. Phys. 55, 241-376 (1992)

49 Sastry, S., Debedenetti, P. G. \& Stillinger, F. H. Signatures of distinct dynamical regimes in the energy landscape of a glass-forming liquid. Nature 393, 554-557 (1998)

50 Mondello, M., Yang, H.-J., Furuya, H. \& Roe, R.-J. Molecular dynamics simulation of atactic polystyrene. 1. Comparison with X-ray scattering data. Macromolecules 27, 3566-3574 (1994).

51 Furuya, H., Mondello, M., Yang, H.-J., Roe, R.-J., Erwin, R. W., Han, C. C. \& Smith, S. D. Molecular dynamics simulation of atactic polystyrene. 2. Comparison with neutron scattering data. Macromolecules 27, 5674-5680 (1994).

52 Roe, R.-J., Mondello, M., Furuya, H. \& Yang, H.-J. Molecular dynamics simulation of atactic polystyrene. 3. Short range order. Macromolecules 28, 2807-2818 (1995).

53 Ayyagari, C., Bedrov, D. \& Smith, G. D. Structure of atactic polystyrene: A molecular dynamics simulation study. Macromolecules 33, 6194-6199 (2000).

54 Song, H.-H. \& Roe, R.-J. Structural change accompanying volume change in amorphous polystyrene as studied by small and intermediate angle X-ray scattering. Macromolecules 20, 2723-2732 (1987). 
55 Xia, X. \& Wolynes, P. G. Fragilities of liquids predicted from the random first order transition theory of glasses. Proc. Natl Acad. Sci. USA 97, 2990-2994 (2000).

56 Dudowicz, J., Freed, K. F. \& Douglas, J. F. The glass transition temperature of polymer melts. J. Phys. Chem. B 109, 21285-21292 (2005).

57 Roland, C. M., Hensel-Bielowka, S., Paluch, M. \& Casalini, R. Supercooled dynamics of glass-forming liquids and polymers under hydrostatic pressure. Rep. Progr. Phys. 68 1405-1478 (2005).
58 Rosamonte's Physical Chemistry Website http://physicalchemistryrosamonte.wordpress $\mathrm{com} /$.

59 Matsuoka, S. Entropy, free volume, and cooperative relaxation. J. Res. Natl Inst. Stand. Technol. 102, 213-228 (1997).

60 Capaccioli, S., Ruocco, G. \& Zamponi, F. Dynamically correlated regions and configurational entropy in supercooled liquids. J. Phys. Chem. B 112 10652-10658 (2008). 